\title{
Improving Modified Grey Relational Method for Vertical Handover in Heterogeneous Networks
}

\author{
Imane Chattate $^{1}$, Mohamed El Khaili ${ }^{2}$, Jamila Bakkoury ${ }^{3}$ \\ Laboratory: Signal, Distributed Systems and Artificial Intelligence (SSDIA) \\ ENSET Mohammedia, Hassan II University of Casablanca, 9167 Morocco
}

\begin{abstract}
With the advent of next-generation wireless network technologies, vertical handover has become indispensable to keep the mobile user always best connected $(\mathrm{ABC})$ in a heterogeneous environment, especially the significant number of multimedia applications that require good quality of service $(\mathrm{QoS})$ for users. To handle this issue, an improvement of modified Grey Relational Analysis (MGRA) to select the AlwaysSuitable-Connection (ASC) network has been proposed. Then, Fuzzy analytic hierarchy process (FAHP) method has been used to determine the weight of criteria. In order to validate our contribution, the proposed method called E-MGRA has been applied to obtain the ranking of suitable network. Finally, a simulation has been presented to demonstrate the performances of our developed approach to reduce the number of handovers compared to the classical method.
\end{abstract}

Keywords-Component; vertical handover; network selection; Quality of Service (QoS); Multi Criteria Decision-Making (MCDM); Grey Relational Analysis (GRA); Fuzzy Analytic Hierarchy Process (FAHP)

\section{INTRODUCTION}

The mobile technology revolution is growing day after day, enabling mobile users to communicate anytime and anywhere. This growth has led mobile operators to propose better bandwidth and increase the capacity of the mobile network that must serve all network customers. Face to these constraints, operators must ensure the development of mobile networks while ensuring a QoS adapted. With the diversity of wireless networking technologies such as $(3 \mathrm{G}, 4 \mathrm{G}$, WLAN, WIFI, and future $5 \mathrm{G}$ networks) [1], vertical handover become a necessity for the mobile terminal to move between the available networks without loss of connection. This step is based on a selection of the best target network in a heterogeneous environment. The selection process involves necessarily the employment of decision-making algorithms such as MultiCriteria Decision-Making algorithms (MCDM). Many types of researches dealt with MCDM algorithms has been applied in different decision-making domains. MCDM is used to select a better network during the vertical handover decision phase, taking into consideration multiple decision criteria.

In the context of MCDM approach we recognize numerous algorithms such as Analytic Hierarchy Process (AHP), Distance to Ideal Alternative (DIA), Analytic Network Process (ANP) to calculate the weight criteria, likewise others algorithms to classifying alternatives suchlike Technique for Order Preference by Similarity to Ideal Solution (TOPSIS), VIseKriterijumska Optimizacija I Kompromisno Resenje (VIKOR), ELimination and Choice Expressing Reality
(ELECTRE), Grey Relational Analysis (GRA). Most of these algorithms realized with $\mathrm{ABC}$ [2] (Always-Best-Connection), articulating on performance to choose the best network destination, but with an inefficiency use of resources. To deal with this problem we suggest an algorithm based on ASC (Always-Suitable-Connection) to employ wireless networking resources effectively.

The goal of this paper is to bring an improvement to network selection decision based on ASC, by enhancing MGRA method. For this reason, we involve the Fuzzy analytic hierarchy process (FAHP) and AHP method to determine the criteria of the decision. Afterward, we apply the enhanced MGRA (E-MGRA) to classify the available network which to bring considerable improvement in terms of QoS and reduce the number of vertical handover in comparison with the traditional method.

The remainder of this paper arranged as follows: Section 2 . presents related works of MCDM algorithms for network selection and describes GRA theory. In Section 3, the mathematical model of FAHP and AHP detailed. The detailed process of our proposed method E-MGRA described in Section 4. Then Section 5 describes the simulations and results to assess our algorithms. Finally, Section 6 concludes this work with some perspectives.

\section{RELATED WORK}

The network selection for next-generation networks has become indispensable in the world of mobile networks; it is a necessary step to ensure a ubiquitous vertical handover with better quality of service $(\mathrm{QoS})$ in a heterogeneous environment. In these recent years, many researchers articulated to propose a better wireless network selection algorithm in a heterogeneous network.

In [3-5], the authors presented a study of vertical handover process with major algorithms, and their different types. Therefore, [6] several researches use received signal strength Indication (RSSI) as an only criterion in the network selection decision. Authors of [7] suggested an algorithm based on averaged RSSI to select optimum network. In fact, using simple RSSI will be incapable to meet the demands of the users at the moment of a handover decision.

Decision making is based on various selection criteria such as bandwidth, delay, user preferences, and packet loss. by dint of this immense number of criteria, the multiple criteria decision-making method (MCDM) is used in the handover decision process. In this regard, [8,9] authors describe and 
compare different multiple attribute decision-making method (MADM) algorithms as like simple additive weighting (SAW), AHP, TOPSIS, ELECTRE, VIKOR.

Although, the most effective algorithms are based on artificial intelligence using the techniques such as neural network [10] and fuzzy logic [11]. Whereas, authors of [12] investigate the use of FHAP and AHP to calculate weighting criteria to resolve the MADM problem. In the same context, Authors [13] suggest an algorithm based on Fuzzy AHP using Battery energy as a new criterion. Otherwise, authors [14] have compared the classical Fuzzy TOPSIS with an enhanced Fuzzy TOPSIS method which demonstrates the performance of the new FE-TOPSIS proposed. However, in [15] explore the entropy weight method and GRA to obtain good performance in a heterogeneous network. Similarly, authors of [16,17] uses both the AHP and FAHP methods to determinate the weight of criteria, then GRA algorithm is applying to obtain the ranking of the candidate networks. As a result, this demonstrates better ranking results obtained by GRA algorithm. Due to the shortcoming of these algorithms, authors of [18,19] developed an algorithm based on modified GRA (MGRA) by adopting ASC which choose the most suitable network during the vertical handover decision phase.

The purpose of this work is to investigate a new method based on MGRA to optimizing the vertical handover problem by improving the network selection decision in a heterogeneous environment.

\section{Multi CRiteria Decision MaKing Methods}

\section{A. AHP}

The Analytic Hierarchy Process (AHP), was proposed by SAATY [20], is an effective process for dealing with complex decision making, when multi-criteria are involved. The procedure defined by AHP consists of building a hierarchy model based on context criteria. Calculating weights requires a pairwise comparison process that refers to responding to a chain of comparisons between the attributes of a pair. The context criteria are evaluated by tuples (see Table 1) to judge the importance of a single criterion in comparison with the other.

In our case, we use AHP to calculate the weight vector $w$, which represents the importance of each metric with respect to different classes of QoS. it represents the results $w_{j}>0$, which provide the weight or importance of the attribute $j^{t h}$ [21], since $\sum_{j=1}^{j=M} w_{j}=1$. The pairwise comparison matrix as formalized in Eq. (1).

$A=\left[\begin{array}{ccc}\tilde{a}_{11} & \cdots & \tilde{a}_{1 n} \\ \vdots & \ddots & \vdots \\ \tilde{a}_{m 1} & \cdots & \tilde{a}_{m n}\end{array}\right]$

TABLE I. DESCRIPTION OF IMPORTANCE SCALE [20]

\begin{tabular}{|l|l|}
\hline Importance scale & Description \\
\hline 1 & Equally important \\
3 & Weakly important one over another \\
5 & Strongly important \\
7 & demonstrated important \\
9 & Extremely important \\
$2,4,6$ & Intermediate values \\
\hline
\end{tabular}

\section{B. FAHP}

The classical AHP method is a technique for analyzing complex decisions. However, some researchers find that it contains some weakness in the AHP method evoked by SAATY, such as Yang and Chen [22,23]. The fuzzy AHP technique is an advanced analytical method developed from the traditional AHP it is a combination of fuzzy logic and linguistic variables. The importance of fuzzy logic is to resolve ambiguities in decision-making problems and the ability to define vague data.

Fuzzy set and linguistic variables: Zadeh [24] introduce fuzzy set theory that addresses uncertainty and vagueness of human thought. It is characterized by its capacity to accredit the degree of adhesion between 0 and 1 by using the "linguistic terms".

Triangular fuzzy numbers (TFN) are exploited to propose fuzzy relative importance $[25,26]$. In this inspection, the evaluation criterion in the judgment matrix and weight vector represented by TFNs, using four real numbers expressed by $\mu A(x)=(a, b, c, d ; w)$ [27], where $\mathrm{a}, \mathrm{b}, \mathrm{c}$ and $\mathrm{d}$ are real values and $0<w \leq 1$ presented in "Fig. 1 " a TFN can be defined as:

$\mu_{A(x)}= \begin{cases}w \frac{x-a}{b-a}, & a<x<b \\ w, & b<x<c \\ w \frac{d-x}{d-c}, & c<x<d \\ 0, & \text { Otherwise }\end{cases}$

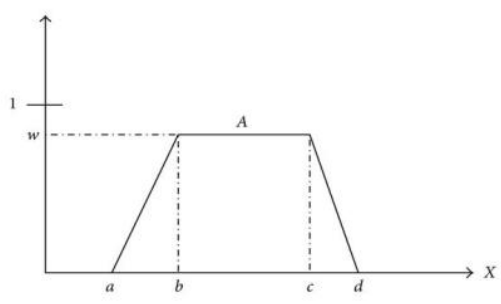

Fig. 1. Trapezoidal Fuzzy Number $\mu_{A(x)}$.

Therefore, we construct a fuzzy pair comparison matrix (Eq (3)), using the trapezoidal fuzzy number (see Table 2), a fuzzy evaluation matrix $Q=\left(q_{i, j}\right)_{n * m}$ is constructed, as: $q_{i, j}=$ $\left(a_{i, j}, b_{i, j}, c_{i, j}, d_{i, j}\right)$ and $q_{i, j}^{-1}=\left(1 / a_{i, j}, 1 / b_{i, j}, 1 / c_{i, j}, 1 / d_{i, j}\right)$.

$A=\left[\begin{array}{ccc}\tilde{a}_{11} & \cdots & \tilde{a}_{1 n} \\ \vdots & \ddots & \vdots \\ \tilde{a}_{m 1} & \cdots & \tilde{a}_{m n}\end{array}\right]$

TABLE II. IMPORTANCE SCALE FOR FUZZY PAIRWISE COMPARISON

\begin{tabular}{|l|l|}
\hline Linguistic Variables & Scale of fuzzy number \\
\hline Very Low (VL) & $(0,0,0.1,0.2)$ \\
\hline Low (L) & $(0.1,0.2,0.2,0.3)$ \\
\hline Medium Low (ML) & $(0.2,0.3,0.4,0.5)$ \\
\hline Medium (M) & $(0.4,0.5,0.5,0.6)$ \\
\hline Medium High (MH) & $(0.5,0.6,0.7,0.8)$ \\
\hline High (H) & $(0.7,0.8,0.8,0.9)$ \\
\hline Very High (VH) & $(0.8,0.9,1.0,1.0)$ \\
\hline
\end{tabular}




\section{A Network SELECTION BASED ON ENHANCED OF MGRA}

\section{A. Modified Grey Relational Analysis (MGRA)}

The grey relational analysis (GRA), introduced by Deng [28], is widely used to solving a variety of multiple decisionmaking problems (MADAM) with uncertain information. The GRA is based on information from the grey system to dynamically compare each attribute quantitatively. This process uses the level of similarity and variability among all the attributes to establish their relationships. The relational analysis proposes how to make predictions and decisions, for the final selection.

Nonetheless, the traditional method GRA is based on an ABC selection, but not an ASC selection, for this fact we will have to consider the ideal values of the parameters as well as the worst parameters. Therefrom we work with a modified method of grey relational analysis (MGRA). The MGRA is used to determine the best available network in a heterogeneous environment, of following the steps above:

- Construction of the decision matrix for the classification of $\mathrm{m}$ networks each having $\mathrm{n}$ attributes of the selection criteria.

$Q_{n, m}=\left(\begin{array}{cccc}q_{11} & q_{12} & \ldots & q_{1 m} \\ q_{21} & q_{22} & \ldots & q_{2 m} \\ \vdots & \vdots & \ddots & \vdots \\ q_{n, 1} & q_{n, 2} & \ldots & q_{n, m}\end{array}\right)$

- Construct normalized decision matrix with values: For benefit attribute,

$r_{i j}=\frac{x_{i j}-\min \left(x_{j}\right)}{\max \left(x_{j}\right)-\min \left(x_{j}\right)}$

For cost attribute,

$r_{i j}=\frac{\max \left(x_{j}\right)-x_{i j}}{\max \left(x_{j}\right)-\min \left(x_{j}\right)}$

- Determination of the ideal and the worst parameters. This step in the classical GRA method determines only the ideal parameters. we define the ideal parameters that represent the maximum values by:

$A^{+}=\left[r_{1}^{+}, r_{2}^{+}, \ldots, r_{m}^{+}\right]$

And the worst parameters that represent the basic requirements by:

$A^{-}=\left[r_{1}^{-}, r_{2}^{-}, \ldots, r_{m}^{-}\right]$

- Calculate Grey Relational Coefficient (GRC) for each of the network with ideal parameters $A^{+}$and worst parameters $A^{-}$as follows:

$$
\begin{aligned}
V_{i j}^{+} & =\frac{\min _{j} \min _{j}\left|r_{i j}-r_{j}^{+}\right|+\mu \max _{i} \max _{j}\left|r_{i j}-r_{j}^{+}\right|}{\left|r_{i j}-r_{j}^{+}\right|+\mu \max _{i} \max _{j}\left|r_{i j}-r_{j}^{+}\right|} \\
V_{i j}^{-} & =\frac{\min _{j} \min _{j}\left|r_{i j}-r_{j}^{-}\right|+\mu \max _{i} \max _{j}\left|r_{i j}-r_{j}^{-}\right|}{\left|r_{i j}-r_{j}^{-}\right|+\mu \max _{i} \max _{j}\left|r_{i j}-r_{j}^{-}\right|}
\end{aligned}
$$

Where $\mu$ is resolution coefficient, $\mu \in[0,1]$, there is always define as 0.5 .

- Consider the weight already calculated by the AHP and FAHP methods mentioned in the previous sections, and the GRC obtained by MGRA with the ideal parameters $A^{+}$and the worst parameters $A^{-}$. Opposed to the traditional method that defines just the ideal parameters in this step.

$r^{+}{ }_{i}=\sum_{j=1}^{n} W_{j} \cdot V^{+}{ }_{i j} \quad \mathrm{i}=1,2 \ldots, n$

Where $\sum_{j=1}^{n} W_{i}=1$

$r^{-}{ }_{i}=\sum_{j=1}^{n} W_{j} \cdot V^{-}{ }_{i j} \quad \mathrm{i}=1,2 \ldots, n$

Where $\sum_{j=1}^{n} W_{i}=1$

- Conclusively, we calculate the decision value $M$ for each of the candidate networks by:

$M_{i}=\frac{\left(r^{+}{ }^{2}\right)^{2}}{\left(r^{+}{ }^{2}\right)^{2}+\left(r^{-}{ }^{2}\right)^{2}} \quad \mathrm{i}=1,2 \ldots, n$

\section{B. Proposed Method E-MGRA}

In order to guide our method towards a selection of the ASC network, and to ensure efficient utilization of resources, where the users' QoS exigency are satisfactory, with a reasonable cost to pay. In this context, we decided to enhance the MGRA method to select the best access network according to the ASC principle. We named the process of our proposed method E-MGRA, starting by modifying the equation (Eq. (13)) of the ancient MGRA method. Previously calculate $\left(r^{+}{ }_{i}\right)^{2} * \mu_{1}$ pursues the properties of the positive solution $\left(r^{+}{ }_{i}\right)^{2}$, of the same $\left(r^{-}{ }_{i}\right)^{2} * \mu_{2}$ pursues the properties of the negative solution $\left(r^{-}{ }_{i}\right)^{2}$. Thus, we construct two relative values $\left(\mu_{1}, \mu_{2}\right)$, respectively to the ideal and worst parameters, then we calculate the new proposed GRC as follows:

$M_{i}=\frac{\left(r^{+}\right)^{2} * \mu_{1}}{\left(r^{+}{ }^{2}\right)^{*} \mu_{1}+\left(r^{-}{ }^{2}\right)^{2} * \mu_{2}} \quad \mathrm{i}=1,2 \ldots, n$

Hence, we mention that the step computes by the equation (Eq. (14)) acquired using the AHP method to achieve the relative importance $\mu_{1}$ and $\mu_{2}$ for each class of traffic. Finally, we choose the network alternatives with the largest $M$ is one of the most suitable for networks users.

\section{Simulation and Results}

\section{A. Simulations}

In the interest of validating the capability of our E-MGRA, we conducted simulation experiments based on AHP used in Section 3.1, and FAHP in Section 3.2 to weight different criteria, the achieved results are also compared.

Foremost, we construct a decision matrix to evaluate alternative networks. Thereafter, the linguistic variable [29] presented in Table 2 is used to create a pairwise comparison matrix using the Fuzzy-AHP method to generate different 
weights. Once the weights are determined, we use the AHP method to determine the relative importance $\mu_{1}$ of the ideal parameter and $\mu_{2}$ of the worst parameters given in Table 3 . Finally, we use our E-MGRA to calculate the new decision value M Proposed (Eq. (14)).

Afterward, we examine E-MGRA method performance on four available networks (LTE (4G), HSPA (3G), WLAN and WiMAX), and we perform the simulation for four classes of QoS traffic (interactive, conversational, background and streaming). During the simulation, we associate for each class of traffic six parameters of different QoS: Throughput $(\mathrm{T})$, Data rate (DR), Jitter (J), Delay (D) and Packet Loss (PL) are randomly varied according to the ranges shown in Table 4 . The simulation repeated for 100 run vertical handover decision points by using MATLAB simulator.

\section{B. Results and Discussion}

In order to compare the effectiveness of our proposed EMGRA method with the MGRA method, we use the average of the number of handovers as well as the ranking abnormality to validate our enhancement, by analyzing the effect of the weight attributed by AHP and FAHP on QoS. The results of the comparison in this paper show that our proposed E-MGRA algorithm outperforms the traditional MGRA algorithm.

TABLE III. THE RELATIVE IMPORTANCE $\mu_{1}$ AND $\mu_{2}$ FOR EACH TRAFFIC Classes [14]

\begin{tabular}{|l|l|l|}
\hline Traffic class & \multicolumn{1}{|c|}{$\mu_{\mathbf{1}}$} & $\mu_{\mathbf{2}}$ \\
\hline Conversational & 0.100 & 0.900 \\
\hline Streaming & 0.250 & 0.750 \\
\hline Interactive & 0.166 & 0.833 \\
\hline Background & 0.125 & 0.875 \\
\hline
\end{tabular}

TABLE IV. THE QOS CRITERIA

\begin{tabular}{|l|l|l|l|l|l|}
\hline Technology & $\begin{array}{l}\text { Throughput } \\
(\mathbf{M b} / \mathbf{s})\end{array}$ & $\begin{array}{l}\text { Data } \\
\text { Rate } \\
(\mathbf{M b} / \mathbf{s})\end{array}$ & $\begin{array}{l}\text { Jitter } \\
(\mathbf{m s})\end{array}$ & $\begin{array}{l}\text { Delay } \\
(\mathbf{m s})\end{array}$ & $\begin{array}{l}\text { Packet } \\
\text { loss }(\%)\end{array}$ \\
\hline LTE (4G) & $0,1-15$ & $30-100$ & $1-6$ & $5-20$ & $25-50$ \\
\hline HSPA (3G) & $1-80$ & $20-80$ & $29-80$ & $2-10$ & $20-80$ \\
\hline WLAN & $1-11$ & $100-$ & $1-12$ & $10-25$ & $90-150$ \\
\hline WiMAX & $1-60$ & $20-90$ & $3-40$ & $3-10$ & $50-120$ \\
\hline
\end{tabular}

Foremost, we present the weights associated with each traffic class with the intention of showing the impact of weights on the ranking of the results of available networks "Fig. 2" exhibit the weights produce by FAHP and AHP methods respectively for Conversational, Background, Streaming, and Interactive traffic classes. In the streaming class, we observe that throughput of the AHP method raised, unlike the other traffic classes whose the packets loss is the uppermost. Correspondingly, we notice that the FAHP method assign weights moderate between all parameters which ensures a balance which generates a better decision.

The average number of handovers is exposed in "Fig. 3" that clarifies the performances of E-MGRA for each class of traffic. We notice that our E-MGRA method overcome the traditional MGRA by reducing the number of handovers with a value of $2 \%$ for conversational, background, and streaming class. Although, interactive traffic dropped by up to $3 \%$ compared to the traditional MGRA algorithm. E-MGRA approach assert that this evolution produces the best selection of network. Finally, we achieve that our proposed approach can effectively solve handover problems in a heterogeneous network.

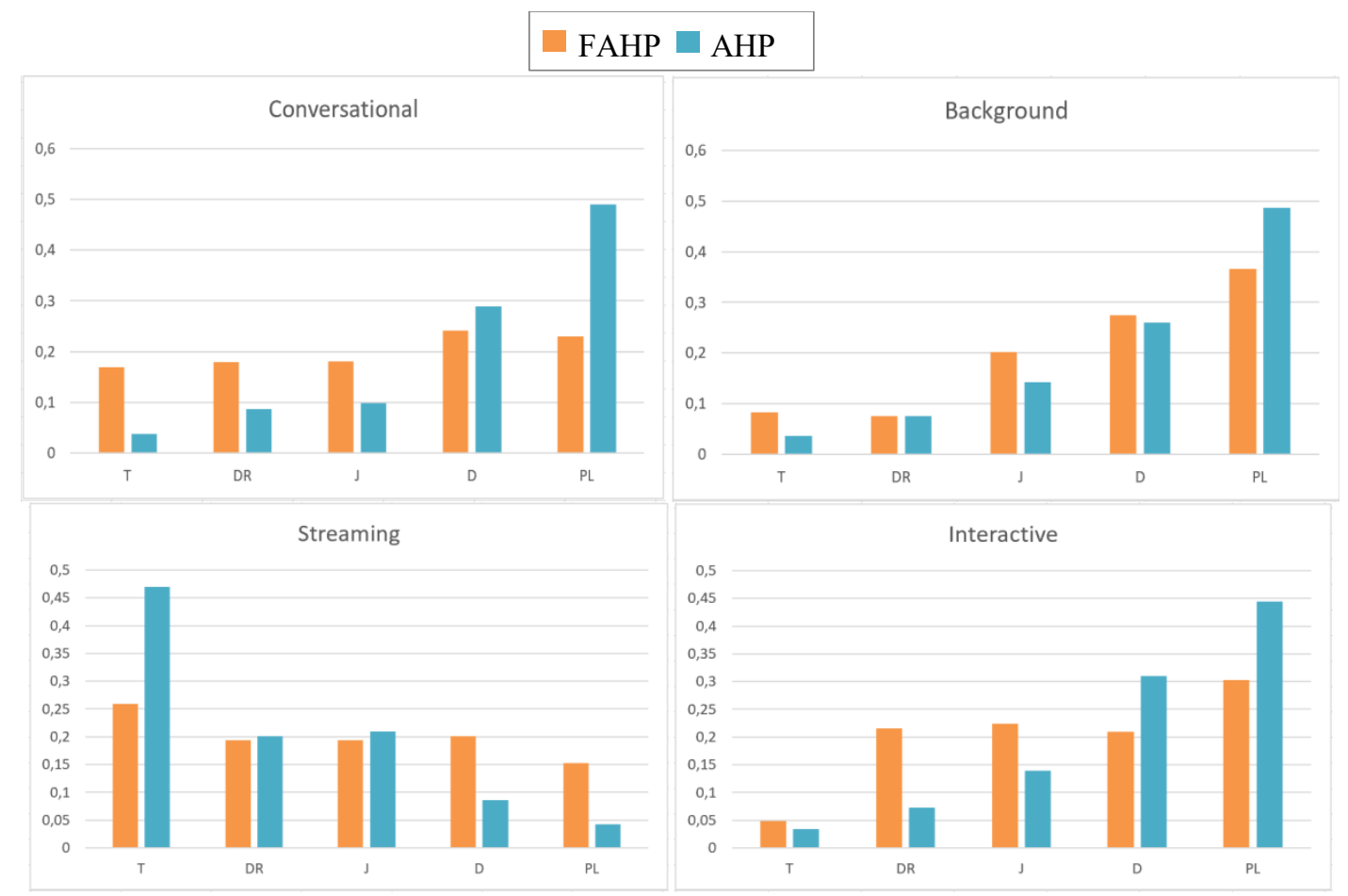

Fig. 2. Associated Weight for Each Class of Traffic. 


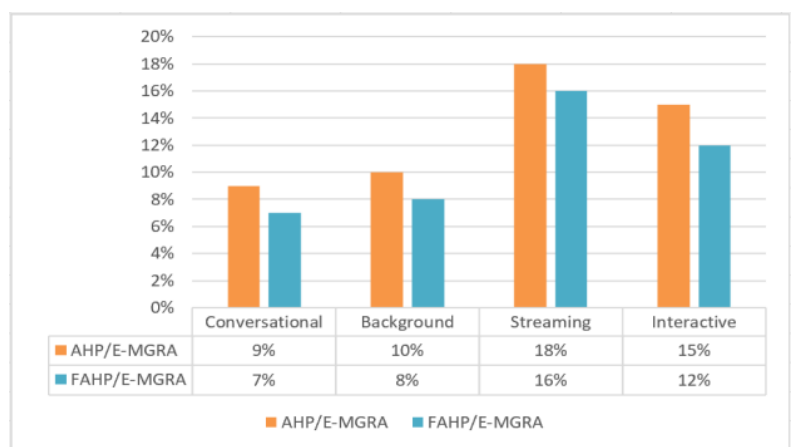

Fig. 3. Average of the Number of Handovers who Proves the Improvement of E-MGRA.

\section{CONCLUSION}

In order to select an optimal network in heterogeneous networks, we propose a new approach which relies on the improvement of MGRA method. Firstly, the AHP and FAHP methods determinate the weight criteria of each QoS class of traffic. After, the enhanced version of MGRA used to select the most suitable network. To validate the performance of the recent E-MGRA method, a comparison of FAHP method with the classical AHP has been performed to produce a fast handover. The results of simulations reveal that our proposed method provides a signification QoS progress for different types of traffic. The E-MGRA method proposed allowed to reduce the number of vertical handovers over the handover execution.

The future research will be focus to combine our proposed method with other MADM methods for making a decision in a heterogeneous network. Moreover, a perspective of this method presented can be efficient in various domains and applications suchlike energy planning, supply chain, and Vehicular ad-hoc networks (VANETs).

\section{ACKNOWLEDGMENT}

This work is supported by the grant of National Center for Scientific and Technical Research (CNRST - Morocco) (No.16UH22016).

\section{REFERENCES}

[1] Gustafsson, Eva, Annika Jonsson, "Always best connected", IEEE Wireless Communications, 2003, 10(1), pp. 49-55.

[2] Omheni, N., Bouabidi, I., Gharsallah, A., Zarai, F., Obaidat, M.S.: Smart mobility management in $5 \mathrm{G}$ heterogeneous networks. IET Netw. 7(3), 119-128 (2018).

[3] I. Chattate, and al. "Overview on technology of vertical handover and MIH architecture". Conference paper-4th IEEE CIST, 2016:31-34.

[4] E. Obayiuwana, and al., "Network selection in heterogeneous wireless networks using multi-criteria decision-making algorithms: a review". In Wireless Networks, pp. 1-33, 2016.

[5] Yan, Xiaohuan, Y. Ahmet Şekercioğlu, and Sathya Narayanan. "A survey of vertical handover decision algorithms in Fourth Generation heterogeneous wireless networks." Computer Networks 54.11 (2010): $1848-1863$.

[6] Xia L., Ling-ge J., Chen H., Hong-wei L. An intelligent vertical handoff algorithm in heterogeneous wireless networks. Int. Con. on Neural Networks and Signal Processing, 2008, pp.550-555.

[7] Ahuja, K., Singh, B., Khanna, R., 2014a. Network selection algorithm based on link quality parameters for heterogeneous wireless networks. Optik - Int. J. Light Electron Opt. 125 (14), 3657-3662.
[8] C.L. Hwang, K. Yoon, Multiple Attribute Decision Making: Methods and Applications, Springer-Verlag, Berlin, 1981.

[9] R. Bikmukhamedov, Y.Yeryomin, J. Seitz, "Evaluation _of MCDAbased Handover Algorithms for Mobile Networks". Ubiquitous and Future Networks (ICUFN), July 2016.

[10] S. Kunarak, R. Sulessathira, "Vertical handover decision management on the basis of several criteria for LVQNN with ubiquitous wireless networks". International Journal of GEOMATE, Vol.12 Issue 34, pp. 123, June 2017.

[11] L. Giupponi, R. Augusti, J. Pérez-Romero, "Sallent O. A novel Joint RadioResource Management Approach with Reinforcement Learning Mechanisms". 24thIEEE International Performance Computing, 2005.

[12] TORFI, F., FARAHANI, R.Z and REZAPOUR, S. (2010), "Fuzzy AHP to determine the relative weights of evaluation criteria and Fuzzy TOPSIS to rank the alternatives", Applied Soft Computing, Vol. 10, No. 2, pp. 520- 528.

[13] CHATTATE, Imane; EL KHAILI, Mohamed; BAKKOURY, Jamila. A Fuzzy-AHP Based Approach for Enhancing Network Selection in Heterogeneous Networks Using Battery Energy Criterion. International Journal of Engineering \& Technology, [S.1.], v. 7, n. 4.32, p. 118-123, dec. 2018.

[14] I.Chattae, M. El Khaili, J.Bakkoury. "A New Fuzzy-TOPSIS based Algorithm for Network Selection in Next-Generation Heterogeneous Networks". Journal of Communications, Vol. 14, No. 3, March 2019.

[15] Sheng, J., Qi, B., Dong, X., Tang, L.: Entropy weight and grey relation analysis-based load balancing algorithm in heterogeneous wireless networks. In: 2012 8th International Conference on Wireless Communications, Networking and Mobile Computing, pp. 1-4 (2012)

[16] Verma, R., Singh, N.P.: GRA based network selection in heterogeneous wireless networks. Wirel. Pers. Commun. 72(2), 1437-1452 (2013).

[17] Xin Song; Wenmin Liu; Minglei Zhang; Feng Liu. A network selection algorithm based on FAHP/GRA in heterogeneous wireless networks. 2nd IEEE International Conference on Computer and Communications (ICCC). 14-17 Oct. 2016.

[18] Wei-wei Jiang; Hong-yan Cui; Qiang-jun Yan; Xiao-juan Wang; JianYa Chen. A Novel Application-Oriented Dynamic Network Selection in an Integrated UMTS and WiMAX Environment. Communications and Networking in China, 2008.

[19] Pei Zhang; Wenan Zhou; Bing Xie; Junde Song. A novel network selection mechanism in an integrated WLAN and UMTS environment using AHP and modified GRA. 2nd IEEE InternationalConference on Network Infrastructure and Digital Content. 24-26 Sept. 2010.

[20] Saaty, R.W., 1987. The analytic hierarchy process-what it is and how it is used. Math. Modelling $9(3), 161-176$.

[21] Saaty, T. L. (1980). The analytical hierarchy process, planning, priority setting, resource allocation. NewYork: Mcgraw Hill.

[22] Ch.Ch. Yang, B.Sh. Chen "Key quality performance evaluation using Fuzzy AHP". Journal of the Chinese Institute of Industrial Engineers, vol. 21(6), pp. 543-550, 2004.

[23] F.T. Bozbura and A. Beskese, "Prioritization of organizational capital measurement indicators using fuzzy AHP", International Journal of Approximate Reasoning, Vol.44, No.2, 2007, pp. 124-147.

[24] L.A. Zadeh, "Fuzzy sets. Information and Control", 8(3), pp. 338-353, 1965.

[25] A. Ishizaka, and P. Nemery, "Multi-criteria decision analysis: methods and software" John Wiley \& Sons, Ltd, Chichester, West Sussex, UK, 2013.

[26] S. H. Chen, "Ranking generalized fuzzy number with graded mean integration" in Proceedings of the 8th International Fuzzy Systems Association World Congress, vol. 2, pp. 899-902, Taipei, Taiwan, 1999.

[27] S. H. Wei and S. M. Chen, "A new approach for fuzzy risk analysis based on similarity measures of generalized fuzzy numbers," Expert Systems with Applications, vol. 36, pp. 589-598, 2009.

[28] Deng, J.L. (1982), "Control problems of grey systems", Systems \& Control Letters, Vol. 1 No. 5, pp. 288-294.

[29] D.Y. Chang "Applications of the extent analysis method on fuzzyAHP”. Eur. J. Oper, Res. 95 ,649-655, 1996. 